\title{
Embedded System Based Radio Detection and Ranging (RADAR) System Using Arduino and Ultra-Sonic Sensor
}

\author{
Ahman Emmanuel Onoja ${ }^{1}$, Abdusalaam Maryam Oluwadamilola ${ }^{1}$, Lukman Adewale AJAO ${ }^{2}$ \\ ${ }^{1}$ Department of Computer Engineering, Federal University of Technology, Minna, Nigeria \\ ${ }^{2}$ Computer Engineering Department, Federal University of Technology, Minna, Nigeria
}

\section{Email address:}

Emmanuel.ahman@st.futminna.edu.ng (A. E. Onoja), Maryam.abdusalaam@st.futminna.edu.ng (A. M. Oluwadamilola)

\section{To cite this article:}

Ahman Emmanuel Onoja, Abdusalaam Maryam Oluwadamilola. Embedded System Based Radio Detection and Ranging (RADAR) System Using Arduino and Ultra-Sonic Sensor. American Journal of Embedded Systems and Applications. Vol. 5, No. 1, 2017, pp. 7-12. doi: 10.11648/j.ajesa.20170501.12

Received: June 30, 2017; Accepted: August 1, 2017; Published: November 28, 2017

\begin{abstract}
The applications of the radio detection and ranging (RADAR) systems in military installations as well as scientific and commercial facilities is powered by the ability of the RADAR systems to use electro-magnetic waves to determine the speed, range, altitude or direction of objects, either fixed or in motion. In the years since RADAR systems came into prominence, incalculable developments have occurred. Some of these are in the fields of navigation and positioning, target detection and tracking, energy optimization, and other applications. In this research, existing radar technologies are examined and an Arduino based RADAR system is proposed. The advantage of this is to drastically reduce power consumption and allow the designers to have access to a wide range of online communities of Arduino programmers and open source reusable code. The system consists of an ultra-sonic sensor, an Arduino micro-controller, a servo motor and a java application for mapping the electro-magnetic waves. A prototype system is built by connecting the ultra-sonic sensors to the Arduino microcontroller's digital input/output pins and the servo motor also connected to the digital input/output pins. Both the ulta-sonic sensor and the servo motor are then clipped together so that as the servo motor sweeps from right to left through an angle of 1800 the servo will rotate alongside it.
\end{abstract}

Keywords: Embedded System, Simulation, Microcontroller, Arduino, Ultra-Sonic Sensors, Servo Motor

\section{Introduction}

All matter produces sound waves just by existing, be it as an effect of the air flow around them their natural frequency. These sound waves can be detected using an ultra-sound sensor. Ultra-sonic waves are sound waves whose frequency are beyond the hearing range of humans $(20,000 \mathrm{~Hz}$ or thereabouts) [4]. The range, $20 \mathrm{~Hz}$ to $20,000 \mathrm{~Hz}$, is essentially the audible spectrum; it varies with individuals and generally reduces with age [5].

An ultra-sonic detector usually consists a transducer to convert electrical signals to sound waves and vice-versa. These have been applied in object detection and identification systems, measuring the orientation and position of objects, collision avoidance systems, surveillance, flow measurement and material type detection [1].

With the linear measurement problem, the introduction of ultrasonic technology provided a much-needed relief as its Ultrasonic Distance Measurer (UDM) allows for non-contact measurement [4]. In this way distances between objects that would otherwise be impossible to measure could be measured.

The speed of travel for sound signals depends proportionally on the square root of the ratio between the medium density and stiffness. This proportionality is a fundamental property of every medium. This property and the speed of sound in air are affected by changes to the environmental conditions like temperature [3].

A common application of ultra-sonic technology is range finding. This is also called sonar which is similar to radar where a pulse of ultra-sound is generated and directed at a particular direction and if there is an object in the path of this sound, it is reflected back to its source and by measuring the time difference between the pulse transmission and reception, it is possible to determine the distance of the object. A typical usage of this method is echo-location by bats [2]. 


\section{Related Literature}

A great many applications have been found for the ultrasonic sensing technology since its emergence [10] and some of these include home security systems, robotics applications, distance measurement, tank level measurement, in production lines, and proximity detection applications [7]. These innumerable applications have made it possible to solve technical problems faster and cheaper without compromising safety, quality and stability [8].

The authors in [9] addressed the use of ultra-sonic sensing capability for home security. The system's design is in such a way that the ultra-sonic sensor is mounted on a servo motor to allow a full $360^{\circ}$ rotations so that in any direction an intruder approaches, the sensor would be able to detect the proximity. Once an intruder is detected, the system automatically emits a beeping sound and following this, an SMS message is sent to the home owner, informing him of an intruder in the house using a GSM module.

The authors in [10] designed a system to aid vehicle drivers to navigate blind spots easily in traffic by installing an ultra-sonic sensor on the vehicle's behind and light on the dashboard to indicate the proximity of other commuters that are coming behind. The sensor is calibrated as a range detecting apparatus and a threshold value set. The driver gets a flash of light on the dashboard when oncoming vehicles become closer than this threshold value.

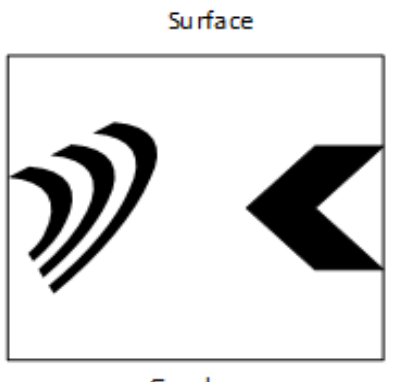

Good
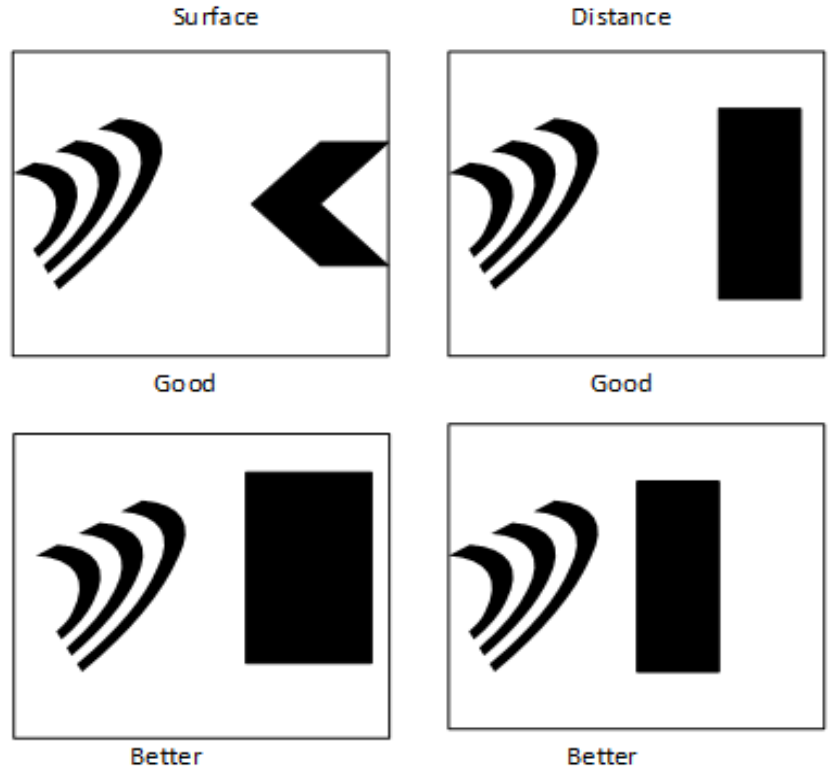

Good

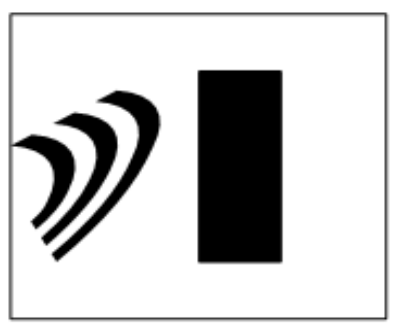

Better
In [11], the authors designed and constructed a navigation application for the visually impaired. The system consisted of an embedded device for detecting local obstacles and an android application for issuing direction to the user verbally. The embedded device uses an ultra-sonic sensor for detecting local obstacles like cars, walls etc. and the data obtained from the ultra-sonic sensor is transmitted to the android application where appropriate decisions are made base on the incoming data and the user is advised accordingly.

The remote monitoring system that was designed and constructed in [12] features the use of ultra-sonic sensors for obstacle avoidance. The system comprises of a remote station (the robot) and a base station (user) who can send commands to the remote station for teleoperation. The remote station comprises of a GPS device, a GSM module, an ultra-sonic sensor-based obstacle avoidance system, a web enabled camera for remote monitoring. Based on the incoming data from the obstacle avoidance system, the GPS system, and the camera, the user is able to send commands to the remote station to alter course or otherwise.

\section{Sound Reflection}

For any meaningful measurement of the distance travelled by sound, the sound needs to be reflected because sound is only reflected when the dimension of the reflective surface is large compared to the wavelength of the sound [2].

Figure 1 illustrates the effect that the dimension of the target surface has on measurement.
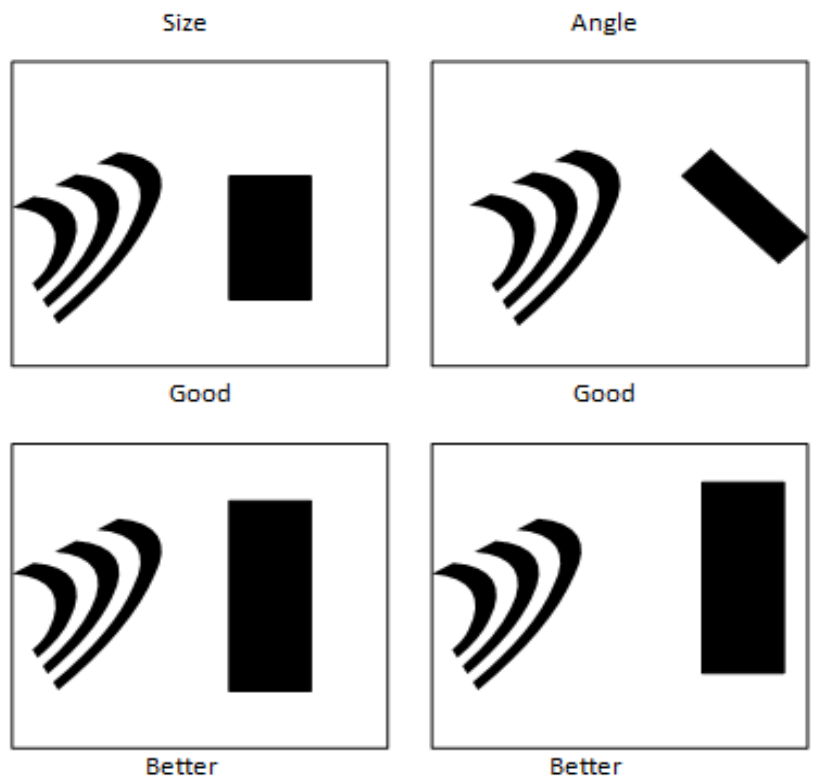

Figure 1. Effect of Surface Dimension on object detection.

\section{Surface}

An ideal target surface is hard and smooth. This surface reflects a greater amount of signal than a soft, rough surface. A weak echo is the result of a small or soft object. This reduces the operating distance of an ultrasonic sensor and decreases its accuracy.

\section{Distance}

The shorter the distance from the ultrasonic sensor to an object, the stronger the returning echo is. Therefore, as the distance increases, the object requires better reflective characteristics to return a sufficient echo.

Angle

The inclination of an objects' surface facing the ultrasonic sensor affects how the object reflects. The portion perpendicular to the sensor returns the echo. If the entire object is at a greater angle, the signal is then reflected away from the sensor and no echo is detected [2-3].

Size 
A large object has more surface area to reflect the signal than a small one. The surface area recognized as the target is generally the area closest to the sensor.

Applications of Sound Reflection

Ultrasonic radar systems were birthed in the defense industry. Because of its myriad applications in under-water object sensing and identification plus identification and tracking of in-coming missiles and vessels [5], the navy and the air force are major names in the RADAR utilization community.

In recent times, applications of this technology have diversified into consumer products such as automatic parking systems, accident prevention systems, and aircraft flight control systems [3].

Another application is in the field of robotics where this technology is now being used by robots as a cheap way to detect, identify and if need be, avoid objects. This is especially effective as robotic home appliances need not have cameras in order to perform their assigned duties like vacuuming, exterminating household pests.

\section{Design and Implementation of RADAR System Using Embedded System Approach}

The development life cycle of the radar project, illustrated in figure 2 shows that it involves the designs of the different components, their implementation, unit testing and finally the integration of the entire system and composite testing. These steps are enumerated thus;

a) Hardware System Design.

b) Hardware Circuit Design.

c) Hardware System implementation.

d) Hardware unit testing.

e) GUI System Design.

f) GUI System Implementation.

g) GUI unit testing.

h) Entire system integration.

i) Entire system testing.

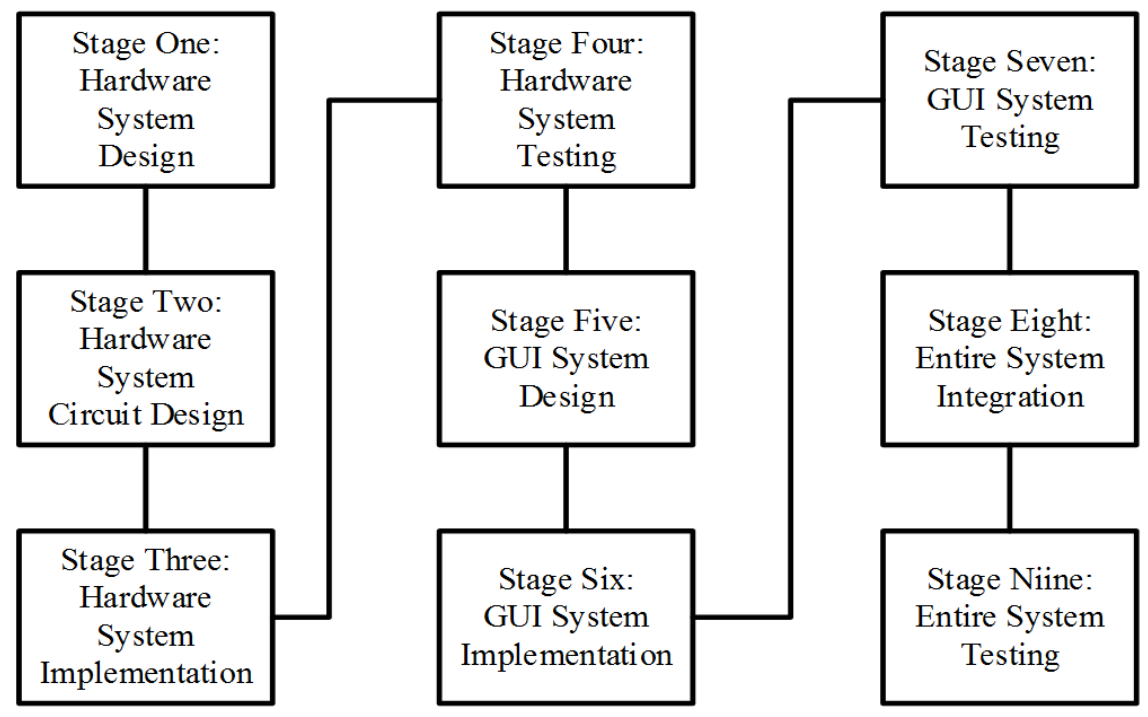

Figure 1. Development life cycle of Radar System.

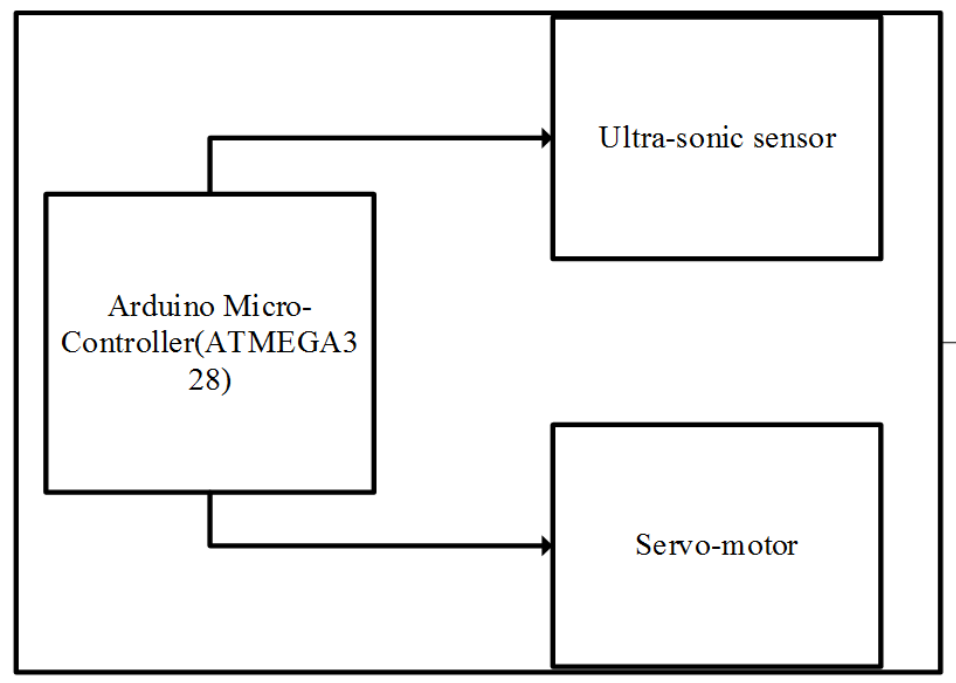

Figure 3. Hardware System Design of Radar System. 


\subsection{Hardware System Design for Radar System}

The hardware system comprises of three distinct components namely, an Arduino micro-controller, a servo motor and an ultra-sonic sensor. The ultrasonic sensor is mounted on the servo-motor to provide a turning mechanism. Both the ultra-sonic sensor and the servo motor are in turn powered and controlled by the Arduino micro-controller. As illustrated in figure 3, both control and power flow from the micro-controller and unto the servo-motor and the ultra-sonic sensor.

\subsection{System Circuit Design}

The hardware system circuit design, shown in figure 4, was designed using fritzing environment. It shows the interconnection between the respective electronic components. In the figure, the trigger pin of the ultra-sonic sensor is connected to the pin D8 of the Arduino microcontroller, the echo pin is connected to pin D7 of the microcontroller and the control line of the servo motor is connected to pin D6 of the micro-controller. The VCC pins of both the servo motor and the ultra-sonic sensor are connected to the $5 \mathrm{~V}$ pinout of the Arduino while the GND pins of both the ultra-sonic sensor and the servo-motor are connected to the GND pinout of the Arduino.

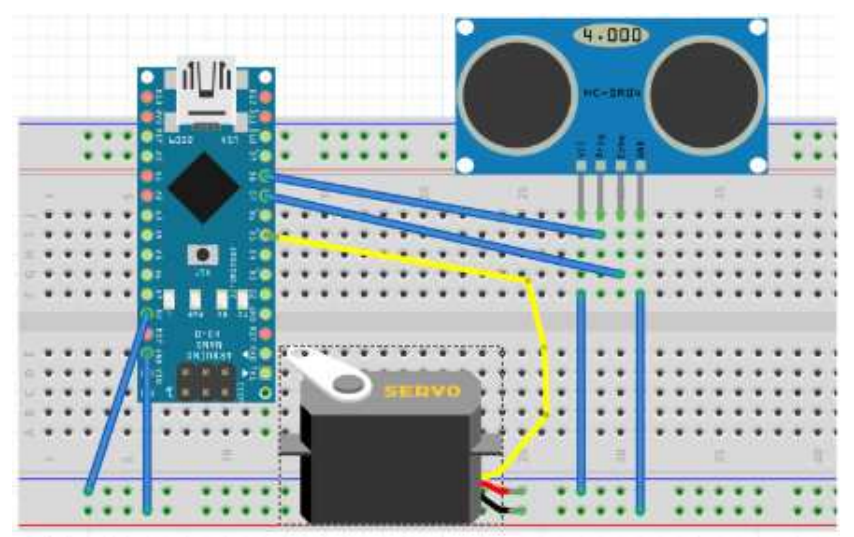

fritzing

Figure 4. Radar hardware sub-system Circuit Diagram on Fritzing.

\subsection{System Circuit Implementation on Breadboard}

Figure 5 shows the complete implementation of the hardware system on a breadboard. It can be seen that the ultra-sonic sensor is mounted on the servo-motor and the entire components are attached to the breadboard using a rubber-band so as to provide extra weight to prevent the entire setup from tipping over when the servo-motor begins to turn.

Using the Arduino IDE, code was written and uploaded to the Arduino micro-controller. The Arduino code was tasked with reading the current position of the servo-motor and posting it to the serial port along with the distance of the nearest object in its path.

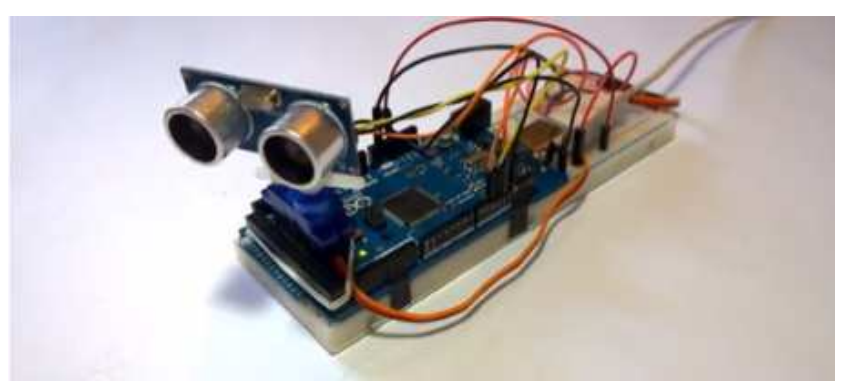

Figure 5. Breadboard of the hardware system implementation.

The Arduino code for the system is highlighted below;

// Includes the Servo library

\#include $<$ Servo.h $>$.

// Defines Tirg and Echo pins of the Ultrasonic Sensor

const int trigPin $=8$;

const int echoPin $=7$;

// Variables for the duration and the distance

long duration;

int distance;

Servo myServo; // Creates a servo object for controlling the servo motor

void $\operatorname{setup}()\{$

pinMode(trigPin, OUTPUT); // Sets the trigPin as an Output

pinMode(echoPin, INPUT); // Sets the echoPin as an Input

Serial.begin(9600);

myServo.attach(6); // Defines on which pin is the servo motor attached

\}

void $\operatorname{loop}()\{$

// rotates the servo motor from 15 to 165 degrees

for(int $\mathrm{i}=15 ; \mathrm{i}<=165 ; \mathrm{i}++)$ \{

myServo.write(i);

$\operatorname{delay}(30)$;

distance $=$ calculateDistance () ;

Serial.print(i); // Sends the current degree into the Serial Port

Serial.print(",");

Serial.print(distance); // Sends the distance value into the Serial Port

Serial.print(".");

\}

// Repeats the previous lines from 165 to 15 degrees

for (int $\mathrm{i}=165 ; \mathrm{i}>15 ; \mathrm{i}--)\{$

myServo.write(i);

$\operatorname{delay}(30)$;

distance $=$ calculateDistance () ; 
Serial.print(i);

Serial.print(",");

Serial.print(distance);

Serial.print(".");

\}

\}

// Function for calculating the distance measured

by the Ultrasonic sensor

int calculateDistance ()\{

digitalWrite(trigPin, LOW);

delayMicroseconds(2);

// Sets the trigPin on HIGH state for 10 micro seconds

digitalWrite(trigPin, $\mathrm{HIGH}$ );

delayMicroseconds(10);

digitalWrite(trigPin, LOW);

duration = pulseIn(echoPin, HIGH); // Reads the echoPin, returns the sound wave travel time in microseconds

distance $=$ duration $* 0.034 / 2$;

return distance; \}

\subsection{Hardware System Testing}

After the hardware system was implemented on the breadboard, a serial cable was used to connect the Arduino to the development machine. From the Arduino IDE, the serial monitor was opened and the results obtained is shown in figure 6.

\begin{tabular}{||l|}
\hline RadarProject \\
\hline \hline \begin{tabular}{l} 
Object \\
\hline $\begin{array}{l}\text { Distance } \\
\text { Angle }\end{array}$ \\
\hline $\begin{array}{l}\text { getD istance } 0 \\
\text { getAngle } 0 \\
\text { mapLocation } 0\end{array}$ \\
\hline
\end{tabular}
\end{tabular}

Figure 7. UML Class Diagram for GUI sub-system

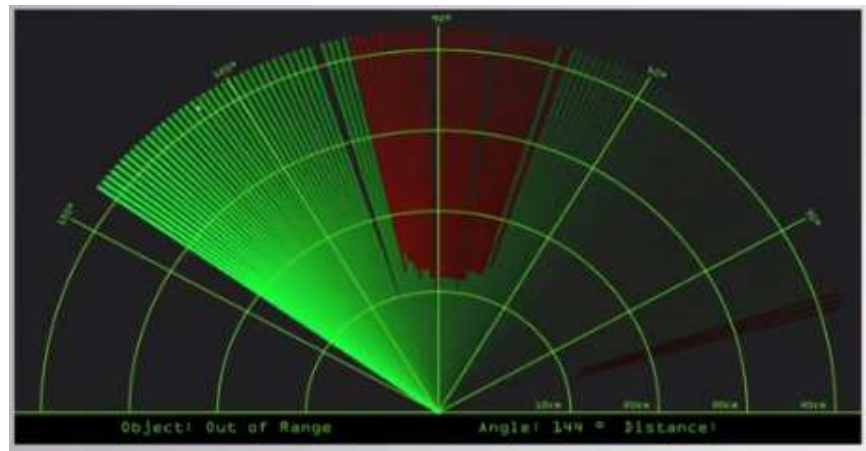

Figure 8. GUI Implementation for the mapping interface.

\section{Conclusion}

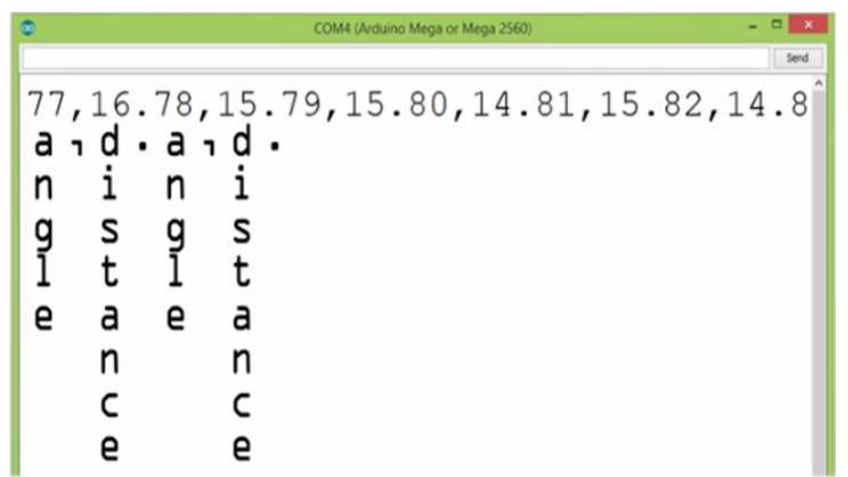

Figure 6. Testing Result of hardware system on Arduino Serial Monitor.

\subsection{GUI System Design and Implementation}

The GUI is built using the JAVA programming language and the package consist of two classes that are represented in the UML class diagram in figure 7.

The Object class of the Radar Project package represents the object that the system encounters in its path and it has attributes like the distance from target/range and the angle/direction where the object is located. Its procedures/methods/functions include the get Distance () method which extracts the distance/range from the serial input stream, the get Angle () method which extracts the angle/direction from the serial input and finally the map Location () method that takes both the angle and distance and maps these coordinates on the GUI.

The final GUI is depicted in figure 8 and a line sweeps from east to west and back and at each point where the ultra-sonic sensor detects an obstacle, a smudge is printed on the GUI.

In this paper, a lab-scaled radar system was designed and implemented using an Arduino, a servo-motor and an ultrasonic sensor. The developed system is able to read the distance of obstacles and the angle of incident and convert this data into visually represented information. The system performance measures up with other systems at its level as it adequately reports any obstacle it finds in its path and provides an estimated range of the object.

A very handy application for this system would be in the area of object detection and avoidance systems for robotics 
or maybe in intrusion detection systems for location sizes where it may not be economical to use multiple units to provide adequate coverage.

The system's range is dependent on the range of the ultrasonic sensor that is used. In this system, the HC-SR04 ultrasonic sensor was used which has a range between $2 \mathrm{~cm}$ and $40 \mathrm{~cm}$.

\section{References}

[1] G. Bhor, P. Bhandari, R. Ghodekar and S. Deshmukh, "Mini Radar," International Journal of Technical Research and Applications, pp. 68-71, 2016.

[2] D. B. Kadam, Y. B. Patil, K. V. Chougale and. S. S. Perdeshi, "Arduino Based Moving Radar System," International Journal of Innovative Studies in Sciences and Engineering Technology (IJISSET), vol. 3, no. 4, pp. 23-27, 2017.

[3] T. P. Rajan, K. K. Jithin, K. S. Hareesh, C. A. Habeeburahman and. A. Jithin, "Range Detection based on Ultrasonic Principle," International Journal of Advanced Research in Electrical, Electronics and Instrumentation Engineering, vol. 3, no. 2, pp. 7638-7643, 2014.

[4] P. S. Abhay,. S. K. Akhilesh, P. Amrit and Kriti, "A Review on Ultrasonic Radar Sensor for Security system," Journal of Emerging Technologies and Innovative Research (JETIR), pp. 137-140, 2016.
[5] P. P. Arun, M. A. Sudhakar, P. MeghaSunil and S. S. Balaji, "Ultrasonic Distance Meter," SVERIAN Scientific, pp. 1-4, 2015.

[6] O. V. Amondi, "Collision Avoidance System," The University Of Nairobi, 2009.

[7] Shamsul A., Tajrian M., "Design of an Ultrasonic Distance Meter", International Journal of Scientific \& Engineering Research, pp. 1-10, March 2013.

[8] U. Papa, G. D. Core, "Design of sonar sensor model for safe landing of an UAV", Proc. IEEE Metrol. Aerosp., pp. 346350, Jun. 2015.

[9] Abbay P., Akhilesh S., Amrit P., and Prof Kriti, "A Review on Ultrasonic Radar Sensor for Security system", Journal of Emerging Technologies and Innovative Research (JETIR), April 2016.

[10] Babu Varghese, "Collision Avoidance System in Heavy Traffic \& Blind spot Assist Using Ultrasonic Sensor", International Journal of Computer Science and Engineering Communications-IJCSEC. Vol. 2, Isuue 1, February, 2014 ISSN: 2347-8586.

[11] S. Bharambe, R. Thakker, H. Patil, K. M. Bhurchandi, "Substitute eyes for blind with navigator using android", Proc. Texas Instrum. India Edu. Conf. (TIIEC), pp. 38-43, Apr. 2013.

[12] D. Sunehra, A. Bano, S. Yandrathi, "Remote monitoring and control of a mobile robot system with obstacle avoidance capability", Proc. Int. Conf Adv. Comput. Commun. Inf. (ICACCI), pp. 1803-1809, Aug. 2015. 